\title{
Group Additivity for Thermochemical Property Estimation of Lignin Monomers on Pt(111)
}

\author{
Supporting Information
}

\author{
Geun Ho Gu and Dionisios G. Vlachos \\ Catalysis Center for Energy Innovation and Center for Catalytic Science and Technology, University of \\ Delaware, Newark, DE 19716 \\ E-mail:vlachos@udel.edu, tel.302-831-2830
}

\section{Adsorption Conformation of Lignin Monomer Ring}

Using the binding type approach, we identified a total of 107 ring conformations (201 including $f c c / h c p$ variation). This section shows chemical drawings of conformations including hydrogenated derivatives of: (1) benzene ring (Figure S1), (2) benzene ring with one H-deficient ring carbon (caused by $\mathrm{C}_{\text {ring }}-\mathrm{O}$ scission) (Figure $\mathrm{S} 2$ ), (3) benzene ring with one surface interacting oxygen substituent (whether $\sigma$ binding or $=\mathrm{O}_{\mathrm{wk}}$ binding) (Figure S3), (4) benzene ring with one H-deficient ring carbon and one surface interacting oxygen substituent (Figure S4), and (5) benzene ring with two surface interacting oxygen substituents (Figure S5). 


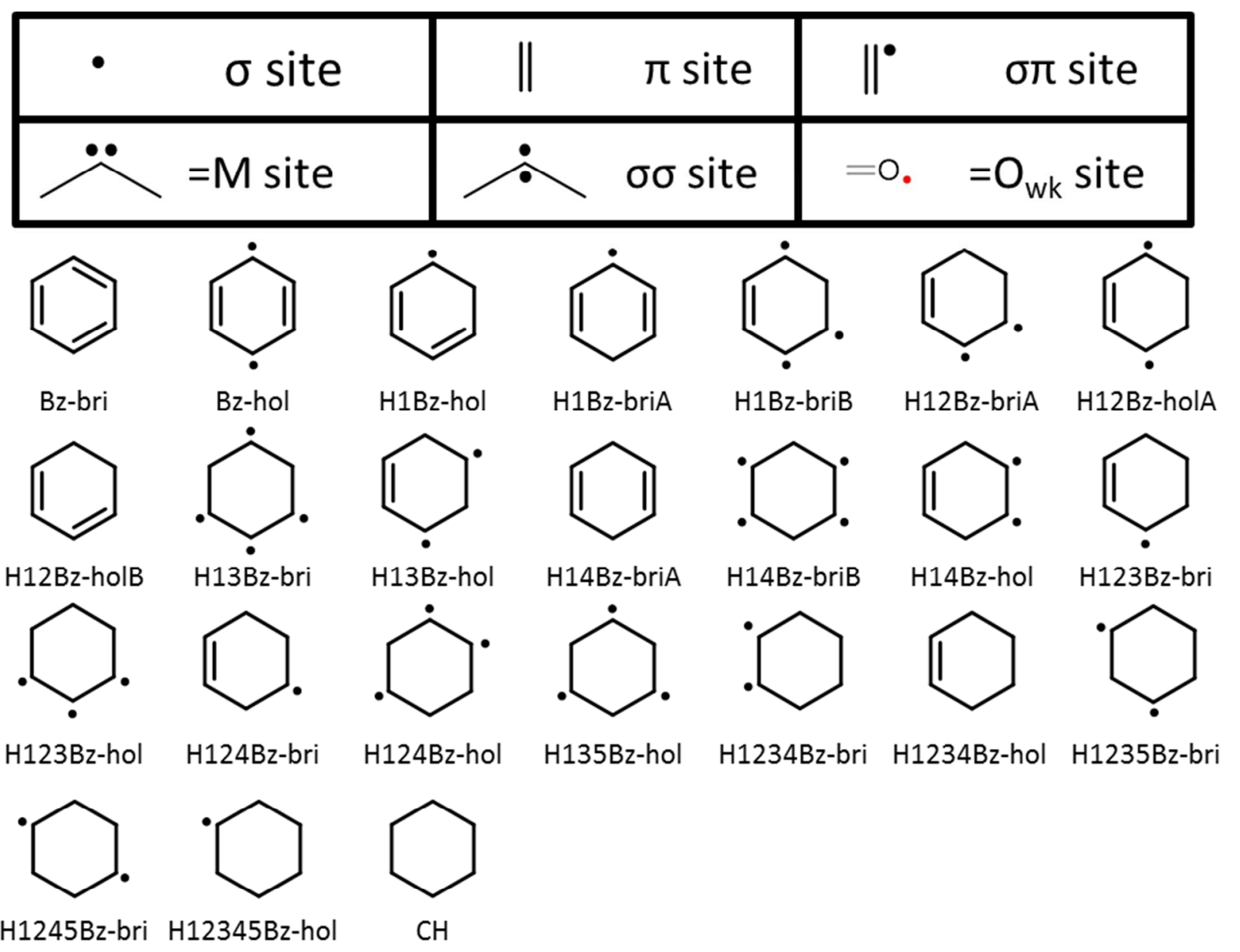

Figure S1. Adsorption conformations of benzene ring and its hydrogenated derivatives. Dots and double bonds represent various binding types of atoms as shown in the legend at the top. ${ }^{1}$ The name convention follows the previous benzene adsorption study by Morin et al. ${ }^{1}$ 
<smiles>[C+]1C=C[CH+]C=C1</smiles><smiles>[C]1C=C[C]C=C1</smiles>

H-1_Bz-1<smiles>[C]1CC=CCC1</smiles>

$\mathrm{H}+1-3 \_\mathrm{Bz}-2$<smiles>[C]1CC=CCC1</smiles>

$\mathrm{H}+12-4$ _Bz-1
H-1_Bz-2<smiles>[C]1C=CCC=C1</smiles>

$\mathrm{H}+1-4$ _Bz-1<smiles>CC1CC=CCC1</smiles>

$\mathrm{H}+12-4$-Bz-2<smiles>[C]1C=CC=CC1</smiles>

H-1_Bz-3<smiles>[C]1C=C[CH-]CC1</smiles>

$\mathrm{H}+1-4 \_\mathrm{Bz}-2$<smiles>[C]1CCCCC1</smiles>

$\mathrm{H}+13-2$ _Bz-1<smiles>[C]1C=CC=CC1</smiles>

$\mathrm{H}+1-2 \_\mathrm{Bz}-1$<smiles>[C]1CC=CCC1</smiles>

H+12-3_Bz-1<smiles>[C+]1CC=CCC1</smiles>

$\mathrm{H}+13-2$-Bz-2<smiles>[C]1CC=CCC1</smiles>

$\mathrm{H}+1-2 \_\mathrm{Bz}-2$<smiles>[C+]1C=C[CH+]CC1</smiles>

$\mathrm{H}+12-3 \_B z-2$<smiles>[C]1CCCCC1</smiles>

$\mathrm{H}+13-4$ _Bz-1<smiles>[C]1C=CCCC1</smiles><smiles>[C]1CCCCC1</smiles><smiles>[C]1CCCCC1</smiles><smiles>[C]1CCCCC1</smiles>

$\mathrm{H}+123-4$ _Bz-1

H+123-4_Bz-2

$\mathrm{H}+123-4$-Bz-3

H+123-5_Bz-1<smiles>[C]1CC=CCC1</smiles><smiles>C[C]1CC[C+]CC1</smiles><smiles>C[C]1CCCCC1</smiles><smiles>C[C]1CC[C]CC1</smiles><smiles>[C]1CCCCC1</smiles><smiles>[C]1=CCCCC1</smiles><smiles>[C]1CCCCC1</smiles>

$\mathrm{H}+124-3 \_B z-1 \quad \mathrm{H}+124-3 \_B z-2 \quad \mathrm{H}+124-5 \_B z-1 \quad \mathrm{H}+124-6 \_B z-1 \quad \mathrm{H}+135-2 \_B z-1 \quad \mathrm{H}+1234-5 \_B z-1 \quad \mathrm{H}+1234-5 \_B z-2$<smiles>CC1CCCCC1</smiles><smiles>C1CCCCC1</smiles><smiles>C1CCCCC1</smiles><smiles>C1CCCCC1</smiles><smiles>C1CCCCC1</smiles>

$\mathrm{H}+1234-5 \_B z-3 \quad \mathrm{H}+1235-4 \_B z-1 \quad \mathrm{H}+1245-3 \_B z-1 \quad \mathrm{H}+12345-6 \_B z-1 \quad \mathrm{H}+12345-6 \_B z-2$

Figure S2. Adsorption conformations of benzene ring with one H-deficient ring carbon and its hydrogenated derivatives. Blue circles indicates Pt atoms. 
<smiles>[O]C1=C[CH+]C=C[CH]1</smiles>

O2_Bz-bri<smiles>O=C1C=CCCC1</smiles>

H+12_O4_Bz-1<smiles>CO[C@@H]1CCC[C@@H](C)C1</smiles>

H+123_O4_Bz-1<smiles>O=C1CCCCC1</smiles>

$\mathrm{H}+1234 \mathrm{O} 5 \mathrm{Bz}-2$<smiles>[O][C]1[C]CC=CC1</smiles>

$\mathrm{H}+1$ _O2_Bz-1<smiles>[O-]C1=CCCC[C]1</smiles>

$\mathrm{H}+12$ _O4_Bz-2<smiles>O=C1CCCCC1</smiles>

H+123_O5_Bz-1<smiles>[O][C]1CCCCC1</smiles>

H+1235_O4_Bz-1<smiles>O=C1CC=CCC1</smiles><smiles>C[C@H]1CC[CH]C(=O)C1</smiles>

H+1_O3_Bz-1<smiles>[O-]C1=C[CH+]CC[CH]1</smiles>

$\mathrm{H}+12$ _O4_Bz-3<smiles>[O]C1CC=CCC1</smiles>

H+124_O3_Bz-1<smiles>CO[C@]1CC[C@](C)CC1</smiles><smiles>[O-]C1=C[CH+]CC[CH]1</smiles>

H+1_O4_Bz-1<smiles>CO[C@@H]1[CH][CH][CH][CH]C1</smiles>

H+13_O4_Bz-1<smiles>C[C@@H]1C[CH]C(=O)CC1</smiles>

H+124_O6_Bz-2<smiles>[O][C]1CC=CCC1</smiles>

$\mathrm{H}+12$ _O3_Bz-1<smiles>CO[C@H]1CC[C@H](C)CC1</smiles>

H+14_O2_Bz-1<smiles>[O][C]1CCCCC1</smiles>

H+1234_O5_Bz-1

Figure S3. Adsorption conformations of benzene ring with one surface interacting oxygen substituent and its hydrogenated derivatives.<smiles>[O-]C1=CCC=C[CH+]1</smiles>

$\mathrm{H}-1$-O2_Bz-bri<smiles>[O]C1CC=CCC1</smiles>

$\mathrm{H}+12-4 \_03 \_\mathrm{Bz}-1$<smiles>CO[C@H]1CC[C@H](O)CC1</smiles><smiles>C[C@H]1CC=C([O-])CC1</smiles>

$\mathrm{H}+1-2 \_03 \_\mathrm{Bz}-1$<smiles>[O-]C1=CCCC[CH+]1</smiles>

H+12-4_O5_Bz-1<smiles>O=C1CCCCC1</smiles><smiles>[O-]C1=CCCC[CH+]1</smiles>

H+1-3_O4_Bz-1<smiles>COC1=CC[C@@H](C)CC1</smiles>

H+13-4_05_Bz-1<smiles>[O-]C1=CCC=C[CH]1</smiles>

H+1-4_O3_Bz-1<smiles>[O][C]1[C]CC[C]C1</smiles>

$\mathrm{H}+13-5 \_04 \_\mathrm{Bz}-1$<smiles>[O-]C1=C[C+]CCC1</smiles>

$\mathrm{H}+12-3$-O4_Bz-1<smiles>[O-]C1=CCCC[CH]1</smiles><smiles>O=C1CCCCC1</smiles>

H+123-4_O5_Bz-1 H+123-5_O4_Bz-1

H+124-5_06_Bz-1 H+1234-5_06_Bz-1

Figure S4. Adsorption conformations of benzene ring with one H-deficient ring carbon and one surface interacting oxygen substituent and their hydrogenated derivatives.<smiles>[O-]C1=C([O-])C[C@@H]2OC3=C([CH]C=C[CH]C3)O[C@@H]2[CH]1</smiles><smiles>[O-]C1=C([O-])CCCC1</smiles><smiles>[O]C1CCCCC1=O</smiles><smiles>O=C1[C]([O-])CCC[C@H]1O</smiles><smiles>O=C1CCCCC1[O-]</smiles><smiles>[O]C1=CCCCC1=O</smiles>

O12_Bz-1 H+12_O45_Bz-2 H+1_O34_Bz-1 H+123_O45_Bz-1 H+12_O34_Bz-1 H+1234_O56_Bz-1 H+12_O45_Bz-1

Figure S5. Adsorption conformations of benzene ring with two surface interacting oxygen substituents and its hydrogenated derivatives. 
Schematics of Binding Type Couples

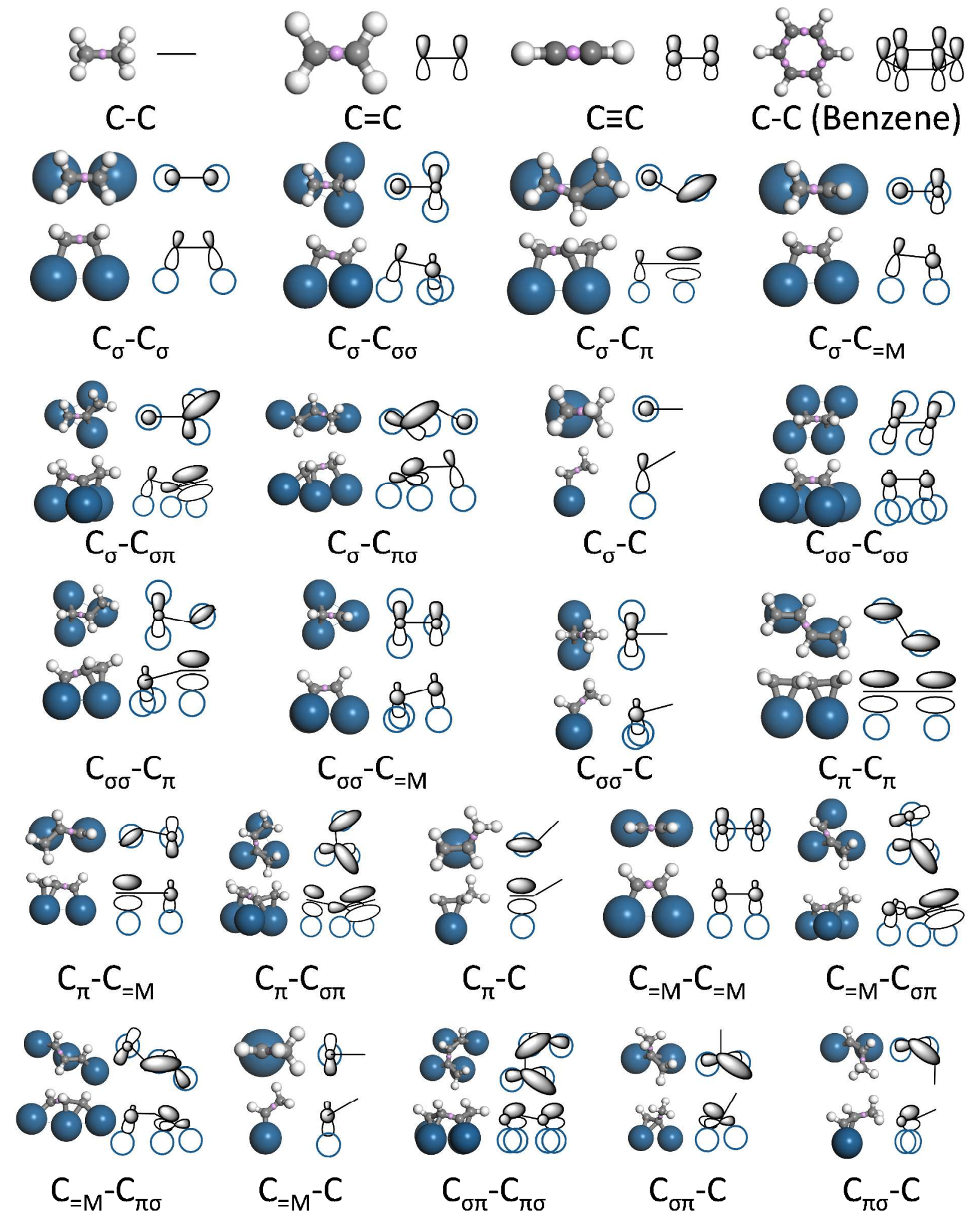

Figure S6. Schematics of binding type couples listed in Table 1 along with expected $p$-orbital lobes in the same phase. ${ }^{2-6}$ BCPs are shown in pink balls and Pt atoms in blue circles. 


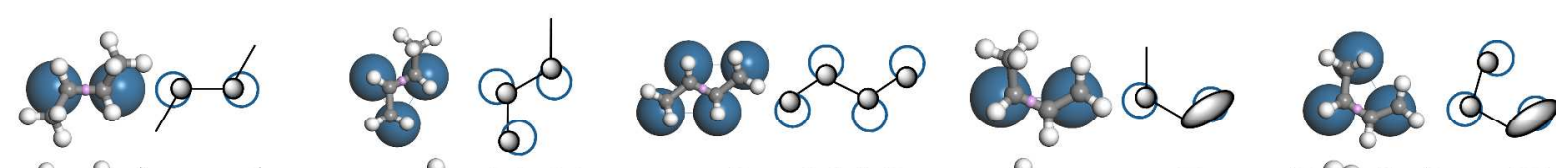

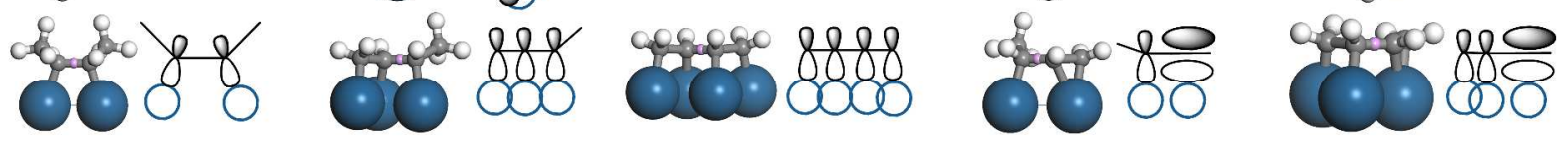
$\begin{array}{lllll}\text { (C) } C_{\sigma}-C_{\sigma}(C) & (C) C_{\sigma}-C_{\sigma}\left(C_{\sigma}\right) & \left(C_{\sigma}\right) C_{\sigma}-C_{\sigma}\left(C_{\sigma}\right) & (C) C_{\sigma}-C_{\pi}\left(C_{\pi}\right) & \left(C_{\sigma}\right) C_{\sigma}-C_{\pi}\left(C_{\pi}\right)\end{array}$

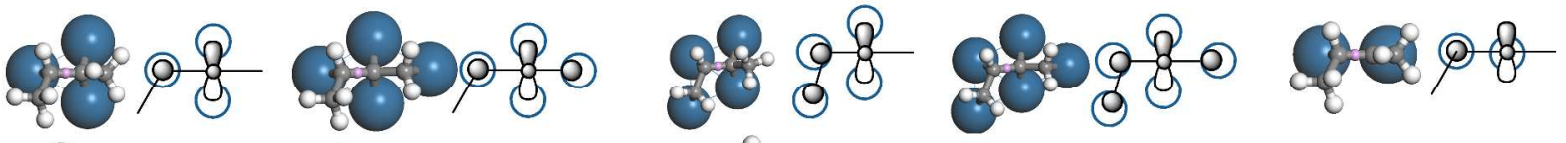

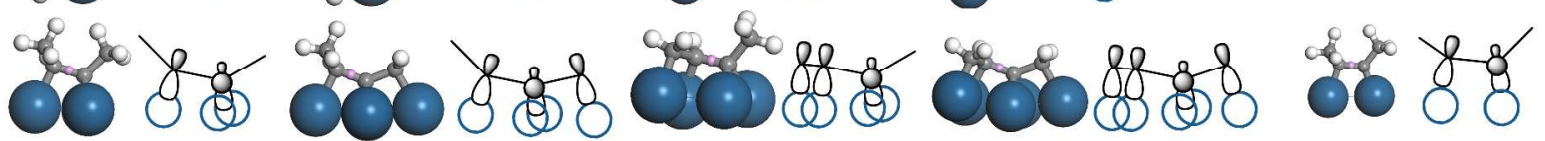
$\begin{array}{lllll}(\mathrm{C}) \mathrm{C}_{\sigma}-\mathrm{C}_{\sigma \sigma}(\mathrm{C}) & (\mathrm{C}) \mathrm{C}_{\sigma}-\mathrm{C}_{\sigma \sigma}\left(\mathrm{C}_{\sigma}\right) & \left(\mathrm{C}_{\sigma}\right) \mathrm{C}_{\sigma}-\mathrm{C}_{\sigma \sigma}(\mathrm{C}) & \left(\mathrm{C}_{\sigma}\right) \mathrm{C}_{\sigma}-\mathrm{C}_{\sigma \sigma}\left(\mathrm{C}_{\sigma}\right) & (\mathrm{C}) \mathrm{C}_{\sigma}-\mathrm{C}_{=\mathrm{M}}(\mathrm{C})\end{array}$

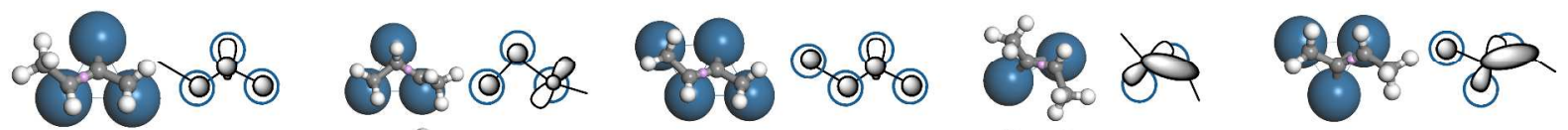

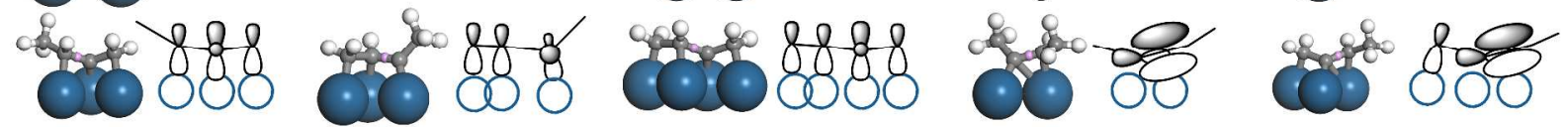
$\begin{array}{lllll}(C) C_{\sigma}-C_{=M}\left(C_{\sigma}\right) & \left(C_{\sigma}\right) C_{\sigma}-C_{=M}(C) & \left(C_{\sigma}\right) C_{\sigma}-C_{=M}\left(C_{\sigma}\right) & (C) C_{\sigma \pi}-C_{\pi \sigma}(C) & \left(C_{\sigma}\right) C_{\sigma \pi}-C_{\pi \sigma}(C)\end{array}$

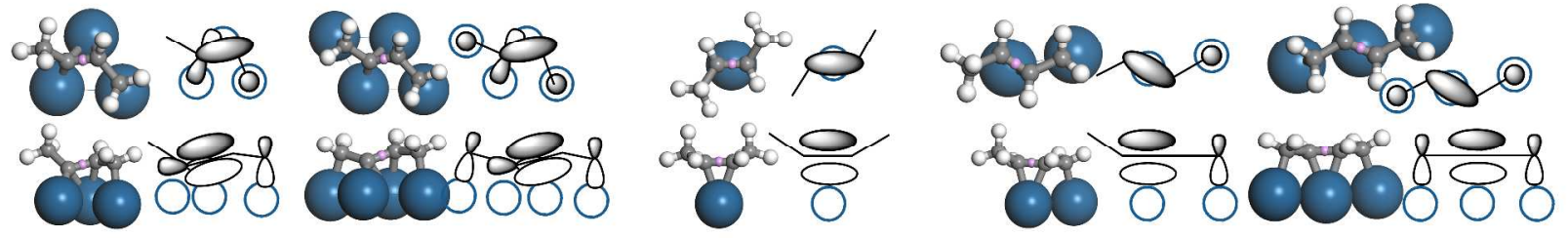
$\begin{array}{lllll}(\mathrm{C}) \mathrm{C}_{\sigma \pi}-\mathrm{C}_{\pi \sigma}\left(\mathrm{C}_{\sigma}\right) & \left(\mathrm{C}_{\sigma}\right) \mathrm{C}_{\sigma \pi}-\mathrm{C}_{\pi \sigma}\left(\mathrm{C}_{\sigma}\right) & (\mathrm{C}) \mathrm{C}_{\pi}-\mathrm{C}_{\pi}(\mathrm{C}) & \left(\mathrm{C}_{\sigma}\right) \mathrm{C}_{\pi}-\mathrm{C}_{\pi}(\mathrm{C}) & \left(\mathrm{C}_{\sigma}\right) \mathrm{C}_{\pi}-\mathrm{C}_{\pi}\left(\mathrm{C}_{\sigma}\right)\end{array}$

Figure S7. Schematics of structures listed in Table 2 along with expected $p$-orbital lobes in the same phase. 


\section{Charge flow Analysis}

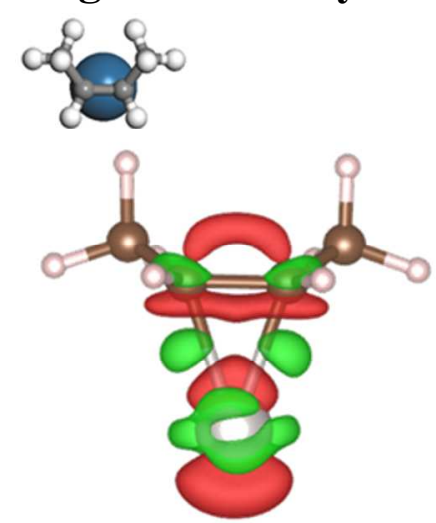

$\pi$
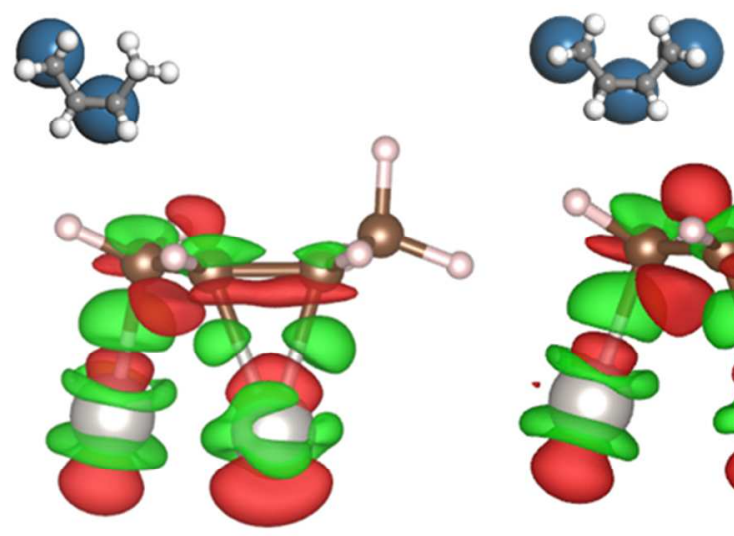

$\sigma-\pi$

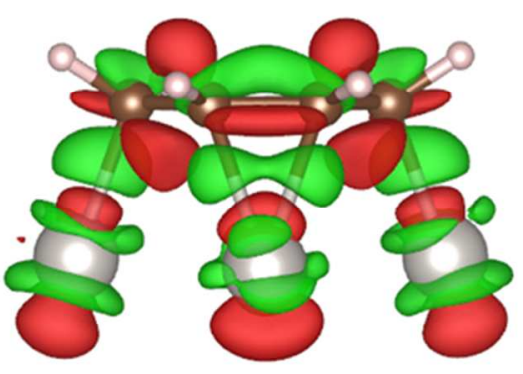

$\sigma-\pi-\sigma$

Figure S8. Electron density difference upon $\pi$-mode adsorption of a $\mathrm{C}_{2} \mathrm{H}_{2}$ fragment. Charge occupation of the conjugated $\pi$ electron system depends on binding of substituent groups.

\section{Methoxy Strain Correction}

In the training set, we observed strong strain related to $-\mathrm{OCH}$ and $-\mathrm{OCH}_{2}$ groups. The strain is visually observable where the methoxy and the ring are pulled towards the bridging oxygen, and the Pt atoms are pulled out of the surface (Figure S9 a, b). These strained fragments involve methoxy group bound to the surface, and the $\alpha$-carbon and the two $\beta$-carbons bound to three separate $\mathrm{Pt}$ atoms (Figure S9 c). We assigned two different corrections for the fragment involving $-\mathrm{OCH}$ and $-\mathrm{OCH}_{2}$ groups. 
(a)
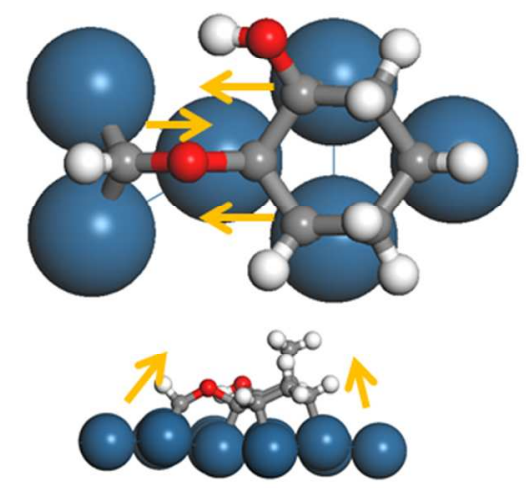

M171

(c)

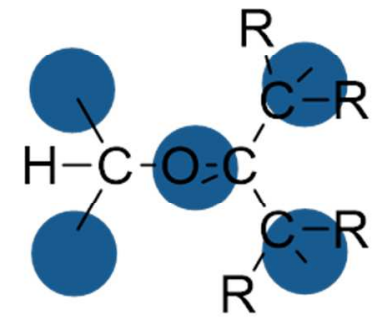

(b)
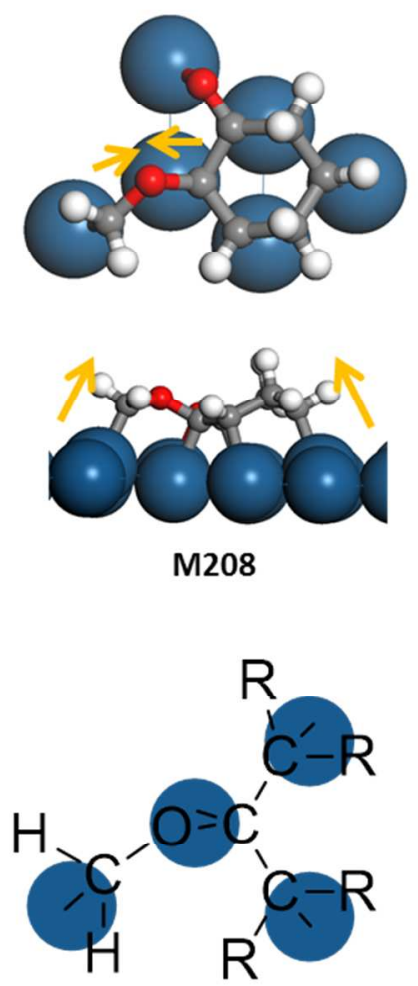

Figure S9. (a) An example of a strained molecule with a-OCH group. (b) An example of a strained molecule with a $-\mathrm{OCH}_{2}$ group. (c) Schematics of strained fragments. 


\section{REFERENCES}

(1) Morin, C.; Simon, D.; Sautet, P. Intermediates in the hydrogenation of benzene to cyclohexene on Pt(111) and Pd(111): A comparison from DFT calculations. Surf. Sci. 2006, 600 (6), 1339-1350.

(2) Calhorda, M. J.; Lopes, P. E. M.; Friend, C. M. Theoretical studies of ethylene adsorption and oxidation on clean and oxygen covered rhodium (111). J. Mol. Catal. A: Chem. 1995, 97 (3), 157-171.

(3) Kobayashi, H.; Kato, H.; Tarama, K.; Fukui, K. A molecular orbital calculation of adsorption of ethylene and acetylene on nickel cluster. J. Catal. 1977, 49 (3), 294-304.

(4) Kokalj, A.; Dal Corso, A.; de Gironcoli, S.; Baroni, S. Adsorption of ethylene on the $\operatorname{Ag}(000$ 1) surface. Surf. Sci. 2002, 507-510, 62-68.

(5) Saeys, M.; Reyniers, M.-F.; Marin, G. B.; Neurock, M. Density Functional Study of Benzene Adsorption on Pt(111). J. Phys. Chem. B 2002, 106 (30), 7489-7498.

(6) Hoffmann, R. Solids and Surfaces: A Chemist's View of Bonding in Extended Structures.; VCH: New York, U.S., 1988. 